\title{
Uma Abordagem usando o Fibrado de Clifford sobre a Geometria Diferencial de Branas
}

\author{
Waldyr Alves Rodrigues Jr., Samuel Augusto Wainer, \\ Instituto de Matemática e Estatística e Computação Científica, IMECC-UNICAMP, \\ 13083-859, Campinas, SP \\ E-mail: walrod@ime.unicamp.br, samuelwainer@ime.unicamp.br
}

Resumo: Primeiramente relembraremos, usando o formalismo do fibrado de Clifford (FFC) de formas diferenciais e a teoria dos extensores agindo em $\mathcal{C} \ell(M, g)$ (o fibrado de Clifford de formas diferencias), a formulação da geometria intrínseca de uma variedade diferenciável $M$ equipada com um tensor métrico $\boldsymbol{g}$ de assinatura $(p, q)$ e uma conexão compatível com a métrica arbitrária $\nabla$, introduzindo o campo $(2-1)$-extensorial de torção $\tau$, o campo $(2-2)$-extensorial de curvatura $\mathfrak{R}$ e (uma vez fixado o calibre) o (1-2)-extensor de conexão $\omega$ e o operador de Ricci $\boldsymbol{\partial} \wedge \boldsymbol{\partial}$ (onde $\boldsymbol{\partial}$ é o operador de Dirac agindo em seções de $\mathcal{C} \ell(M, g)$ ) o qual apresenta grande importância nesse trabalho. Em seguida, usando o FFC daremos uma apresentação da geometria Riemanniana ou Lorentziana de uma subvariedade orientável $M(\operatorname{dim} M=m)$ mergulhada em uma variedade $\stackrel{\circ}{M}$ (tal que $\stackrel{\circ}{M} \simeq \mathbb{R}^{n}$ está equipada com uma métrica semi-Riemanniana $\stackrel{\mathrm{g}}{ }$ de assinatura $(p, q), \quad p+q=n$ e com conexão de Levi-Civita $\stackrel{\circ}{D})$ onde definimos uma métrica

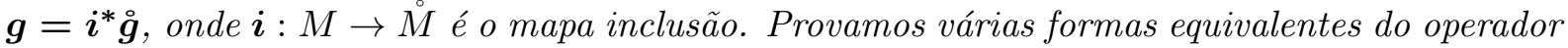
de curvatura $\mathfrak{R}$ de $M$ [11]. Mostraremos um resultado muito importante, de que o operador de Ricci de $M$ é o (negativo) quadrado do operador de formato (shape operator do inglês) $\mathbf{S}$ de $M$ (objeto obtido aplicando-se o operador projeção $\mathbf{P}$ à restrição sobre $M$ do operador de Dirac $\partial$ de $\mathcal{C} \ell(\stackrel{\circ}{M}, \stackrel{\circ}{g}))$. Também obteremos a relação entre o $(1-2)$-extensor de conexão $\omega$ e a biforma de formato (do inglês shape biform) $\mathcal{S}$ (um objeto relacionado com $\mathbf{S}$ ). Os resultados obtidos são usados para dar uma formulação matemática para a teoria de Clifford da matéria. Esperamos que nosso trabalho seja útil para geômetras diferenciais e físicos teóricos interessados, e.g., em teoria de cordas e branas e na teoria da relatividade, divulgando e expandindo resultados muito importantes que aparecem na referência [5].

Palavras-chave: Fibrado de Clifford, Branas, Relatividade Geral, Operadores de Formato, Operador Curvatura, Operador de Ricci, Tensor de Curvatura

\section{Introdução}

Neste trabalho usamos o formalismo do fibrado de Clifford (FFC) para analisarmos a geometria Riemanniana e Lorentziana de uma subvariedade orientável $M(\operatorname{dim} M=m)$ mergulhada numa variedade $\stackrel{\circ}{M}$ tal que $\stackrel{\circ}{M} \simeq \mathbb{R}^{n}$ está equipada com uma métrica semi-Riemanniana $\stackrel{g}{\text { g com }}$ assinatura $(p, q)$ e $p+q=n)$ e sua conexão de Levi-Civita $\stackrel{\circ}{D}$. Os resultados que citamos nessa seção podem ser encontrados em [11].

Para atingirmos nossos objetivos e exibirmos alguns resultados interessantes que não são bem conhecidos (e os quais, e.g.,possivelmente podem ser do interesse para a descrição e formulação das teorias de branas [7] e teorias de cordas $[1,4]$ ) primeiramente relembraremos como formular usando FFC a geometria intrínseca de uma estrutura $\langle M, \boldsymbol{g}, \nabla\rangle$ onde $\nabla$ é uma conexão geral de Riemann-Cartan compatível com a métrica, i.e., $\nabla \boldsymbol{g}=\mathbf{0}$ e os tensores de Riemann e de torção de $\nabla$ são não nulos. Na nossa abordagem introduziremos (desde de que fixado um calibre no fibrado das bases) um campo $(1,2)$-extensorial $\boldsymbol{\omega}: \sec \bigwedge^{1} T^{*} M \rightarrow \sec \bigwedge^{2} T^{*} M$ intimamente 
relacionado com a 1-forma de conexão que permite escrever uma fórmula muito interessante para a derivada covariante para qualquer seção do fibrado de Clifford da estrutura $\langle M, \boldsymbol{g}, \nabla\rangle$. Será mostrado que $\boldsymbol{\omega}$ está relacionado com $\mathcal{S}: \sec \bigwedge^{1} T^{*} M \rightarrow \sec \bigwedge^{2} T^{*} M$ a biforma de formato da variedade.

Suporemos que $M$ é uma subvariedade própria $^{1}$ de $\stackrel{\circ}{M}$ na qual qual $\boldsymbol{i}: M \mapsto \stackrel{M}{M}$ é o mapa inclusão. Introduzindo coordenadas naturais globais $\left(\boldsymbol{x}^{1}, \ldots, \boldsymbol{x}^{n}\right)$ para $\stackrel{M}{2} \simeq \mathbb{R}^{n}$ escrevemos $\stackrel{\mathrm{g}}{=}$ $\sum_{i, j=1}^{n} \eta_{i j} d \boldsymbol{x}^{i} \otimes d \boldsymbol{x}^{j} \equiv \eta_{i j} d \boldsymbol{x}^{i} \otimes d \boldsymbol{x}^{j}$ e equipamos $M$ com a métrica pullback $\boldsymbol{g}:=\boldsymbol{i}^{*} \stackrel{\boldsymbol{g}}{ }$. Nós então encontramos a relação entre a conexão de Levi-Civita $D$ de $\boldsymbol{g}$ e $\stackrel{\circ}{D}$, a conexão de Levi-Civita $\stackrel{\boldsymbol{g}}{\text {. }}$ Suporemos que $\boldsymbol{g}$ é não degenerado de assinatura $(p, q)$ com $p+q=m$.

$\mathcal{C} \ell(\stackrel{\circ}{M}, \stackrel{\mathrm{g}}{)})$ e $\mathcal{C} \ell(M, \mathrm{~g})$ denotam respectivamente o fibrado de Clifford de formas diferenciais de $\stackrel{\circ}{M}$ e $M^{2}$. No que segue $\stackrel{\circ}{g}=\sum_{i, j=1}^{n} \eta^{i j} \frac{\partial}{\partial \boldsymbol{x}^{i}} \otimes \frac{\partial}{\partial \boldsymbol{x}^{j}} \equiv \eta^{i j} \frac{\partial}{\partial \boldsymbol{x}^{i}} \otimes \frac{\partial}{\partial \boldsymbol{x}^{j}}$ é a métrica de fibrado cotangente.

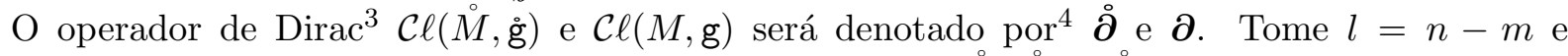
$\left\{\stackrel{\circ}{e}_{1}, \stackrel{\circ}{e}_{2}, \ldots, \stackrel{\circ}{e}_{\boldsymbol{m}}, \stackrel{\circ}{e}_{m+1}, \ldots, \stackrel{\circ}{e}_{m+l}\right\}$ uma base ortonormal para $T \stackrel{\circ}{U}(\stackrel{\circ}{U} \subset M)$ tal que $\left\{e_{\mathbf{1}}, e_{\mathbf{2}}, \ldots, e_{\mathbf{m}}\right\}=$

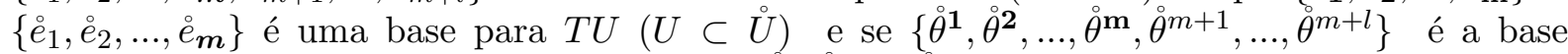
dual de $\left\{e_{i}\right\}$ teremos que $\left\{\theta^{\mathbf{1}}, \theta^{\mathbf{2}}, \ldots, \theta^{\mathbf{m}}\right\}=\left\{\dot{\theta}^{1}, \AA^{2}, \ldots, \theta^{m}\right\}$ é uma base para $T^{*} U$ dual à base $\left\{e_{\mathbf{1}}, \boldsymbol{e}_{\mathbf{2}}, \ldots, e_{\mathbf{m}}\right\}$ de $T U$. Teremos, como é bem conhecido [12]:

$$
\stackrel{\check{\partial}}{=} \sum_{i=1}^{n} \stackrel{\circ}{\theta}^{i} \stackrel{\circ}{D}_{\boldsymbol{e}_{i}}=\stackrel{\circ}{ }^{i} \stackrel{\circ}{D}_{\boldsymbol{e}_{i}}, \quad \boldsymbol{\partial}=\sum_{i=1}^{m} \theta^{i} D_{\boldsymbol{e}_{i}}=\theta^{\mathbf{i}} D_{\boldsymbol{e}_{\mathbf{i}}},
$$

Note que usamos os índices sub e sobrescritos em negrito para denotarmo as bases $\left\{e_{\mathbf{i}}\right\}$ e $\left\{\theta^{\mathbf{i}}\right\}$ do espaço tangente e cotangente de $M$. Esta notação é convenientemente usada neste trabalho.

A base dual à base coordenada natural $\left\{\frac{\partial}{\partial \boldsymbol{x}^{i}}\right\}$ será denotada no que segue por $\left\{\gamma^{i}\right\}$ onde, $\gamma^{i}=$

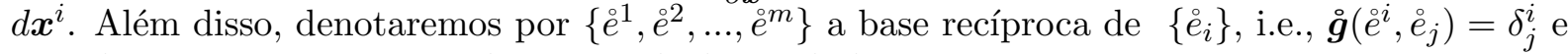
por $\left\{\stackrel{\circ}{\theta}_{i}\right\}$ a base recíproca de $\left\{\stackrel{\circ}{\theta}^{i}\right\}$, i.e., $\stackrel{\circ}{g}\left(\stackrel{\circ}{\theta}^{i}, \stackrel{\circ}{\theta}_{j}\right):=\stackrel{\circ}{\theta}^{i} \cdot \stackrel{\circ}{\theta}_{j}=\delta_{j}^{i}$. Também note que para $\mathbf{i}, \mathbf{j}=1, \ldots, m$ vale $\mathrm{g}\left(\theta^{\mathbf{i}}, \theta_{\mathbf{j}}\right)=\stackrel{\mathrm{g}}{\mathrm{g}}\left(\stackrel{\circ}{ }^{i}, \stackrel{\circ}{j}_{j}\right)$. Escreveremos também $\mathrm{g}\left(\theta^{\mathbf{i}}, \theta_{\mathbf{j}}\right)=\theta^{\mathbf{i}} \cdot \theta_{\mathbf{j}}=\delta_{\mathbf{j}}^{\mathbf{i}}$. A representação do operador de Dirac $\stackrel{\partial}{\partial}$ na base coordenada natural de $\stackrel{\circ}{M}$ é, $\sum_{\mathfrak{i}=1}^{\mathfrak{n}} \gamma^{i} \frac{\partial}{\partial \boldsymbol{x}^{i}}={ }_{i=1}^{n} \stackrel{\circ}{ }^{i} \stackrel{\circ}{D}_{\boldsymbol{e}_{i}}$. Note que teremos $\left.\stackrel{\circ}{\theta}^{m+1}\right|_{M}=0, \ldots,\left.\stackrel{\circ}{\theta}^{m+l}\right|_{M}=0$, i.e., para qualquer campo vetorial $\boldsymbol{a} \in \sec T U$ e $d=1, \ldots, l$ teremos

$$
\left.\stackrel{\circ}{\theta}^{m+d}\right|_{M}(\boldsymbol{a})=0 .
$$

Denotaremos também

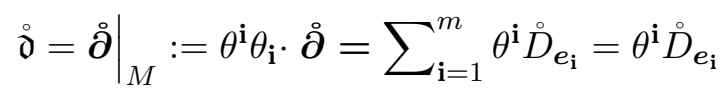

a restrição de $\partial$ na subvariedade $M$. O operador projeção $\mathbf{P}$ (um campo extensorial ${ }^{5}$ ) em $M$, o operador de formato $\mathbf{S}=\mathfrak{d} \mathbf{P}: \sec \mathcal{C} \ell(M, \stackrel{\circ}{\mathrm{g}}) \rightarrow \sec \mathcal{C} \ell(M, \mathrm{~g})$ e a biforma de formato da variedade $M, \mathcal{S}: \sec \bigwedge^{1} T^{*} M \mapsto \sec \bigwedge^{2} T^{*} M, \mathcal{S}(a):=-\left(a \cdot \mathfrak{d} I_{m}\right) I_{m}^{-1}$ (onde $\tau_{\boldsymbol{g}}=I_{m}=\theta^{1} \theta^{2} \cdots \theta^{m}$ é a forma volume ${ }^{6}$ em $\left.U \subset M\right)$ são objetos fundamentais neste estudo.

\footnotetext{
${ }^{1}$ Por uma subvariedade própria (ou regular [2]) $M$ de $\stackrel{\circ}{ }$ nos referimos a um subconjunto $M \subset M$ tal que para

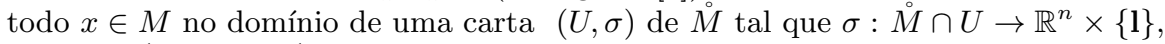
$\sigma(x)=\left(x^{1}, \cdots, x^{n}, l^{1}, \cdots, l^{m-n}\right)$, onde $\mathbf{l} \in \mathbb{R}^{n-m}$.

${ }^{2}$ Para as aplicações do trabalho note que $\bigwedge T^{*} M=\bigoplus_{r=0}^{n} \bigwedge^{r} T^{*} M \hookrightarrow \mathcal{C} \ell(M, \mathrm{~g})$, onde o símbolo $\hookrightarrow$ significa que para cada $x \in M, \wedge T_{x}^{*} M$ (o fibrado das formas diferenciais) está mergulhado em $\mathcal{C} \ell\left(T_{x}^{*} M, \mathrm{~g}_{x}\right)$ e $\bigwedge T_{x}^{*} M \subseteq$ $\mathcal{C} \ell\left(\bigwedge T_{x}^{*} M, \mathrm{~g}_{x}\right)$.

${ }^{3}$ Note que o operador de Dirac usado nesse trabalho age em seções do fibrado de Clifford. Não confundir com o operador de Dirac que age em seções do fibrado Espinorial (veja detalhes em [8]). Este último operador pode ser usado para examinar a topologia da brana, como mostrado em [9]

${ }^{4}$ Seguiremos aqui a notação usada em [12]. Aqui, diferentemente das referências [5, 6, 13], usaremos os operadores contrações à esquerda e à direita $\lrcorner \mathrm{e}\llcorner\mathrm{e}$ o operador produto escalar (denotado por · ) agindo em seções do fibrado de Clifford. Também nossas convenções para o tensor de Riemann fazem com que algumas equações apareçam com sinais diferentes daquelas aparecendo nas referências já citadas.

${ }^{5}$ Para uma apresentação da teoria dos campos extensoriais, veja, e.g., [12].

${ }^{6} \mathrm{~A}$ forma volume $\tau_{\mathrm{g}}$ para $\stackrel{\circ}{U} \subset \stackrel{\circ}{M}$ será denotada por $I_{n}=\check{\boldsymbol{\theta}}^{1} \grave{\boldsymbol{\theta}}^{2} \cdots \stackrel{\leftrightarrow}{\boldsymbol{\theta}}^{m}$. A forma volume $\tau_{\mathrm{g}}$ em $\stackrel{\circ}{U} \subset M$ será denotada por $I_{n}=\check{\theta}^{1} \dot{\theta}^{2} \ldots \check{\theta}^{\mathbf{m}} \dot{\theta}^{m+1} \ldots \stackrel{\circ}{\theta}^{m+l}=I_{m} \stackrel{\circ}{ }^{\mathbf{m}} \dot{\theta}^{m+1} \ldots \stackrel{\circ}{\theta}^{m+l}$.
} 
Nos dedicamos a encontrar várias expressões equivalentes para a biforma de curvatura $\mathfrak{R}(u, v)$ em termos do operador de formato. Relembraremos que a ação do quadrado do operador de Dirac $\boldsymbol{\partial}$ em seções do fibrado de Clifford tem duas decomposições diferentes

$$
\partial^{2}=-(d \delta+\delta d)=\boldsymbol{\partial} \cdot \boldsymbol{\partial}+\boldsymbol{\partial} \wedge \boldsymbol{\partial},
$$

onde $d$ e $\delta$ são respectivamente a derivada exterior e a coderivada de Hodge e $\boldsymbol{\partial} \cdot \boldsymbol{\partial}, \boldsymbol{\partial} \wedge \boldsymbol{\partial}$ são respectivamente o Laplaciano covariante e o operador de Ricci. As formas explícitas de $\boldsymbol{\partial} \cdot \boldsymbol{\partial}$ e $\boldsymbol{\partial} \wedge \boldsymbol{\partial}$ são dadas em [12] onde é mostrado que $\boldsymbol{\partial} \wedge \boldsymbol{\partial}$ é um operador extensorial e o resultado notável

$$
\partial \wedge \partial \theta^{i}=\mathcal{R}^{i}
$$

onde os objetos $\mathcal{R}^{i}=R_{j}^{i} \theta^{j} \in \sec ^{1} T^{*} M \hookrightarrow \sec \mathcal{C} \ell(\stackrel{\circ}{M}, \stackrel{\circ}{\mathrm{g}})$ com $R_{j}^{i}$ as componentes do tensor de Ricci associado com $D$ são chamadas de campos de 1-formas de Ricci. Uma das principais propostas deste trabalho é dar uma prova detalhada da notável equação

$$
\partial \wedge \partial(v)=-\mathbf{S}^{2}(v)
$$

que nos diz que operador de formato é a raiz negativa do operador de $\mathrm{Ricci}^{7}$.

\section{Sobre os pequenos picos de Clifford}

Podemos pensar que do fato de que $\boldsymbol{\partial} \wedge \boldsymbol{\partial}(v)=\mathcal{R}(v)=-\mathbf{S}^{2}(v)$ quando pensada à luz da Relatividade Geral junto com a teoria de branas nos permite dar uma formalização matemática à intuição de Clifford $^{8}$ apresentada em [3], que diz:

(1) As pequenas porções do espaço são de fato de uma natureza análoga a pequenos picos em uma superfície na média chata; de forma que as leis ordinárias da geometria não são válidas neles.

(2) Que esta propriedade de curvatura ou distorção é continuamente passada de uma porção do espaço para outra como uma onda.

(3) Que esta variação da curvatura do espaço é o que realmente acontece no fenômeno que chamamos de movimento da matéria.

(4) Que no mundo físico nada mais acontece além dessa variação, sujeito (possivelmente) à leis de continuidade.

Vejamos como proceder. Seja ${ }^{9}\left(M, \boldsymbol{g}, D, \tau_{g}, \uparrow\right)$ um modelo de um campo gravitacional gerado por um tensor energia momento $T^{\mathbf{a}}:=T_{\mathbf{b}}^{\mathbf{a}} \theta^{\mathbf{a}} \otimes \theta^{\mathbf{b}}$ descrevendo toda matéria do universo de acordo com a teoria da Relatividade Geral. Como é bem conhecido a equação de Einstein pode ser escrita como

$$
\partial \wedge \partial \theta^{\mathbf{a}}=-\mathcal{T}^{\mathbf{a}}+\frac{1}{2} \mathcal{T} \theta^{\mathbf{a}},
$$

onde $\mathcal{T}^{\mathbf{a}}:=T_{\mathbf{b}}^{\mathbf{a}} \theta^{\mathbf{b}}$ and $\mathcal{T}:=T_{\mathbf{a}}^{\mathbf{a}}, \operatorname{com} T_{\mathbf{b}}^{\mathbf{a}}$. Se supusermos que a estrutura $(M, \boldsymbol{g})$ é uma subvariedade de $\left(\stackrel{\circ}{2} \simeq \mathbb{R}^{n}, \stackrel{g}{\mathrm{~g}}\right)$ para $n$ grande o suficiente podemos escrever a Eq.(6) como

$$
\mathbf{S}^{2}\left(\theta^{\mathbf{a}}\right)=\mathcal{T}^{\mathbf{a}}-\frac{1}{2} \mathcal{T} \theta^{\mathbf{a}}
$$

\footnotetext{
${ }^{7}$ Este resultado aparece (com o sinal positivo no segundo membro da Eq.(5) em [5]. Veja também [13]. Entretanto, leve em conta que os métodos utilizados nestas referências usam a álgebra de Clifford de multivetores e assim, comparações com os resultados lá obtidos com as apresentações padrões de geometria diferencial moderna usando formas diferenciais não são nada óbvias, esta seja provavelmente a razão do porquê desses importantes e bonitos resultados apresentados em [5] foram infelizmente ignorados.

${ }^{8}$ Levando-se em conta, é claro, que diferentemente da ideia de Clifford, ao invés de uma teoria espacial da matéria, precisamos falar numa teoria espaço-tempo da matéria.

${ }^{9} \mathrm{O}$ símbolo $\uparrow$ significa que a variedade Lorentziana $(M, \boldsymbol{g})$ é orientada no tempo. Detalhes em [12].
} 
Então, numa região onde não há matéria $\mathbf{S}^{2}\left(\theta^{\mathbf{a}}\right)=0$, apesar do fato de que $\mathbf{S}\left(\theta^{\mathbf{a}}\right)=\mathcal{S}\left(\theta^{\mathbf{a}}\right)$ pode ser não nulo. Então, um ser vivendo num hiper-espaço $\mathbb{R}^{n}$ e olhando para nossa branamundo verá que os pequenos picos (i.e., "matéria") são formatos especiais em $M$, locais onde $\mathbf{S}^{2}\left(\theta^{\mathbf{a}}\right) \neq 0$.

\section{Uma Equação tipo Maxwell para um Brana-Mundo com um Campo Vetorial de Killing}

Quando $(M, \boldsymbol{g})$ admite um campo vetorial de Killing $\boldsymbol{A} \in \sec T M$ então segue de [10] que $\delta A=0$, onde $A=\boldsymbol{g}(\boldsymbol{A},) \in \sec ^{1} T^{*} M \hookrightarrow \sec \mathcal{C} \ell(M, \mathrm{~g})$. Neste caso podemos mostrar que o operador de Ricci aplicado à $A$ é igual a operdor D'Alembertiano covariante aplicado à $A$, i.e.,

$$
\partial \wedge \partial A=\partial \cdot \partial A
$$

Agora, com a Eq.(3) na qual o quadrado do operador de Dirac $\partial^{2}$ pode ser decomposto de duas maneiras, conseguimos,

$$
\partial \wedge \partial A+\partial \cdot \partial A=\partial^{2} A=-d \delta A-\delta d A
$$

Por fim, escrevendo $F=d A$ e levando em conta que $\delta A=0$ a equação de Einstein pode ser reescrita como

$$
\delta F=2 \mathbf{S}^{2}(A)
$$

e desde que $d F=d d A=0$ podemos escrever a equação de Einstein como:

$$
\partial F=-2 \mathbf{S}^{2}(A)
$$

A Eq.(11) nos mostra que numa brana Lorentzian $M$ de dim 4 a qual contém um campo vetorial de Killing $\boldsymbol{A}$, a equação de Einstein é codificada num "campo tipo eletromagnético" $F$ tendo como fonte uma corrente $J=-2 \mathbf{S}^{2}(A) \in \sec \mathcal{C} \ell(M, \mathrm{~g})$.

\section{Conclusões}

Neste trabalho, damos uma apresentação da geometria de variedades usando o formalismo do fibrado de Clifford, com a esperança de prover uma referência útil para pessoas (que conhecem a teoria de Cartan de formas diferencias) ${ }^{10}$ e que estão interessadas na geometria diferencial de subvariedades $M$ de uma variedade $M \simeq \mathbb{R}^{n}$. Provamos em detalhes diversas expressões equivalentes para a biforma de curvatura $\mathfrak{R}(u \wedge v)$ e além disso provamos que o operador de Ricci $\boldsymbol{\partial} \wedge \boldsymbol{\partial}$ quando aplicado a um campo de 1-formas $v$ é tal que $\boldsymbol{\partial} \wedge \boldsymbol{\partial}(v)=\mathcal{R}(v)=-\mathbf{S}^{2}(v)$ $\left(\mathcal{R}(v)=R_{\mathbf{b}}^{\mathbf{a}} \theta_{\mathbf{b}}\right)$ é o negativo do quadrado do operador de formato $\mathbf{S}$. Mostramos que quando este resultado é aplicado à Relatividade Geral permite nos dar uma realização matemática da teoria de Clifford da matéria. Também mostramos que numa brana Lorentziana contendo um campo de vetores de Killing, a equação de Einstein pode ser codificada numa equação tipo Maxwell cuja fonte é uma corrente dada por $J=2 \mathbf{S}^{2}(A)$.

Para finalizar observamos que embora alguns (mas não todos) resultados neste trabalho aparecem em [5, 6, 13], nossa metodologia e muitas provas diferem consideravelmente. Usamos o fibrado de Clifford de formas diferenciais $\mathcal{C} \ell(M, \mathrm{~g})$ ) e demos provas detalhadas para todas as fórmulas, deixando claro importantes issues, apresentando, e.g., a relação precisa entre a biforma de formato $S$ avaliada em $v$ (um campo de 1-formas) e o extensor de conexão $\omega$ avaliado em $v$. Em particular, nossa abordagem também generaliza para uma conexão geral de Riemann-Cartan os resultados em [6] os quais são válidos apenas para conexão de Levi-Civita $D$ de uma métrica Lorentziana de assinatura $(1,3)$.

\footnotetext{
${ }^{10}$ Isto inclui pessoas interessadas em teoria de cordas e branas e Relatividade Geral.
} 


\section{Referências}

[1] Becker, K., Becker M., and Schwarz, J., "String Theory and M-Theory", Cambridge Univ. Press, Cambridge, 2007.

[2] Choquet-Bruhat, Y, DeWitt-Morette, C. and Dillard-Bleick, M., "Analysis Manifold and Physics. Part 1: Basics (revised edition)", North Holland, Amsterdam, 1982.

[3] Clifford, W. K., On the Space-Theory of Matter, Proc. Cambridge Phil. Soc. 2, 157-158 (1864-1876 -Printed 1876).

[4] Duff, M., M-Theory (The theory Formely Known as Strings), Int. J. Mod. Phys. A 11, (1996) 5623-5642.

[5] Hestenes, D., and Sobczyk, G., "Clifford Algebra to Geometric Calculus", D. Reidel Publ. Co., Dordrecht, 1984.

[6] Hestenes, D., Curvature Calcualtions with Spacetime Algebra, Int. J. Theor. Phys. 25, (1986) 581-588.

[7] Mannhein, P. D., "Brane Localized Gravity", World Sci. Publ. Co., Singapore (2005).

[8] Notte-Cuello, E., Rodrigues, W. A. Jr, and Q. A. G. de Souza, The Square of the Dirac and spin-Dirac Operators on a Riemann-Cartan Space(time), Rep. Math. Phys.60, (2007) $135-157$.

[9] da Rocha, R., Bernardini, A. E., and Hoff da Silva, J. M., Exotic Dark Spinor Fields, JHEP 4, article:110 [arXiv:1103.4759] [hep-th] (2011).

[10] Rodrigues, W. A. Jr., Killing Vector Fields, Maxwell Equations and Lorentzian Spacetimes, Adv. Appllied. Clifford Algebras 20, (2010) 871-884.

[11] Rodrigues, W. A. Jr., Wainer, S. A., A Clifford Bundle Approach to the Differential Geometry of Branes, Advances in Applied Clifford Algebras 24, (2014) 617-847.

[12] Rodrigues, W. A. Jr. and Capelas de Oliveira, E., "The Many Faces of Maxwell Equations. A Clifford Bundle Approach", Lecture Notes in Physics 722, Springer, Heildeberg, 2007. Errata and preliminary version of a second edition at http://www.ime.unicamp.br/ ${ }^{\sim}$ walrod/recentes.htm

[13] Sobczyk, G., Conformal Mappings in Geometric Algebra, Not. Am. Math. Soc. 59, (2012) 264-273. 